\title{
Avaliação da eficiência produtiva de hospitais do SUS de Santa Catarina, Brasil
}

\author{
Evaluation of productive efficiency in the Unified \\ National Health System hospitals in the \\ State of Santa Catarina, Brazil
}

\author{
1 Programa de Pós-graduação \\ em Saúde Pública, \\ Universidade Federal \\ de Santa Catarina, \\ Florianópolis, Brasil. \\ 2 Programa de Pós-graduação \\ em Engenharia de \\ Produção, Universidade \\ Federal de Santa Catarina, \\ Florianópolis, Brasil. \\ Correspondência \\ M. C. M. Calvo \\ Departamento de Saúde \\ Pública, Programa de \\ Pós-graduação em Saúde \\ Pública, Universidade \\ Federal de Santa Catarina. \\ Campus Universitário \\ Trindades/n,Florianópolis, SC \\ 88040-900, Brasil. \\ mcmcalvo@ccs.ufsc.br \\ mcmcalvo@terra.com.br
}

\begin{abstract}
This study evaluated the productive efficiency of 112 hospitals under the Unified National Health System (SUS) in the State of Santa Catarina, Brazil. The objective was to verify which hospitals apply the available resources efficiently. The research was based on data from 2003 collected from the SUS Hospital Data System (SIH-SUS). Data Envelopment Analysis (DEA) was applied under the assumption of variable returns to scale. The study focused exclusively on general hospitals with similar characteristics in terms of size and specialization. The results identified 23 efficient hospitals and efficiency targets for each hospital. According to the empirical model, the number of hospitalizations with discharges could be increased by 15\%. Application of an input reduction model would result in savings of $25 \%$ on human resources (physicians and nurse technicians), 17\% on hospital beds, and 13\% on admissions costs for the overall hospital system.
\end{abstract}

Health Evaluation; Organizational Efficiency; Health Services Evaluation
André Cesconetto 1

Jair dos Santos Lapa 2

Maria Cristina Marino Calvo 1

\section{Introdução}

As internações hospitalares do Sistema Único de Saúde (SUS) estão concentradas em unidades conveniadas, onde o sistema público não possui grande capacidade de controle e intervenção. São muitos hospitais privados e universitários onde o controle de gastos públicos é realizado de maneira indireta, pela limitação do número de internações e pela definição dos custos por tipo de procedimento. Existe nos hospitais uma grande oportunidade de maximização do lucro aos prestadores de serviços privados e também de maximização do orçamento aos prestadores de serviços públicos, devido ao atual modelo de pagamento dos serviços prestados. Isso é alcançado ao se empregar técnicas, equipamentos e profissionais altamente especializados em internações mais complexas e onerosas.

Os serviços de saúde devem ser eficientes macroeconomicamente (controle dos custos) e microeconomicamente (maximização dos serviços prestados, maximização da satisfação dos usuários e minimização dos custos). É importante que se tenha como proposta para a utilização dos recursos públicos as premissas de maximização dos resultados com recursos fixos ou minimização dos recursos com resultados pré-determinados.

De acordo com os dados do Sistema de Informação e Orçamento Público em Saúde do Departamento de Informática do SUS (SIOPS/ 
DATASUS; http://siops.datasus.gov.br, acessado em 01/Ago/2005), em 2003 foram realizadas no Brasil cerca de 12 milhões de internações hospitalares, correspondendo a um custo total de 5,9 bilhões de Reais. Isso representou um custo médio de $\mathrm{R}$ \$ 484,64 por internação. Tendo em vista a crescente demanda pelos serviços hospitalares e o montante de recursos repassados pelo SUS, é primordial que seja otimizada a alocação desses recursos.

A avaliação de serviços de saúde é, em geral, efetuada com o uso de indicadores parciais, os quais permitem apenas uma avaliação fracionada da estrutura. Alguns desses indicadores são muito importantes para a gestão dos serviços de saúde, principalmente para estruturas hospitalares. Pode-se citar como exemplo de indicadores importantes a taxa de mortalidade hospitalar e a taxa de ocupação de leitos, as quais permitem uma avaliação imediata de algumas características do hospital.

A situação do Brasil é similar à de muitos outros países que, preocupados com a crescente demanda por recursos de saúde, necessitam estabelecer prioridades e políticas para controle dos gastos. O custo médio das internações é cem vezes maior que o custo médio dos atendimentos ambulatoriais. Dessa forma, o impacto sobre estudos das redes hospitalares no controle e melhor alocação de recursos públicos com a assistência à saúde deve ser maior que estudos similares efetuados com dados dos atendimentos ambulatoriais 1 .

O método mais tradicional de se medir desempenho de uma organização é por meio do cálculo da produtividade parcial (PP), em que um único produto é relacionado a um único insumo. Um exemplo dessa produtividade parcial pode ser o número de consultas realizadas por médico em determinada unidade de atenção básica à saúde. Esse tipo de medida é calculado pela fórmula 1:

$\mathrm{PP}$ = quantidade gerada de produto/quantidade consumida de insumo (1).

Conhecer apenas a produtividade parcial de um hospital não permite saber se o mesmo está operando de maneira eficiente ou não. Já a Fronteira de Produção é constituída pela observação dos Planos de Operação e cada um destes planos corresponde à associação entre quantidades de insumos e quantidades de produtos envolvidos em uma dada operação produtiva.

Quando se emprega a Fronteira de Produção para avaliação de eficiência produtiva, as organizações são divididas em dois grupos: (i) organizações eficientes, que são aquelas associadas aos planos de operação situados na Fronteira de Produção, e suas respectivas produtividades parciais observadas são iguais à máxima produtividade observada ( $\left(\mathrm{PP}^{*}\right)$ e, (ii) organizações ineficientes, que são aquelas cujos planos de operação não estão associados a pontos pertencentes à Fronteira de Produção, ou seja, operando com produtividade parcial menor que PP*.

O nível de ineficiência de uma organização é medido pela distância de seu plano de operação para a Fronteira de Produção. Assim, se a distância de um dado plano de operação para a fronteira de produção é igual a zero é porque este plano de operação está na Fronteira de Produção. A Figura 1 mostra um grupo aleatório de hospitais e suas produtividades parciais.

O segmento de reta W1W2 definido pelos planos de operação W1 e W2 é a referência que torna o hospital $\mathrm{W}$ eficiente, que poderia gerar mais altas com a mesma quantidade de insumo empregada ou manter a atual produção (número de altas) reduzindo a quantidade de insumo empregada, se estivesse localizado em qualquer ponto deste segmento.

A produtividade parcial não considera todos os fatores de produção e isto pode levar a uma interpretação incorreta, por atribuir a um insumo o acréscimo produtivo que pode ter sido gerado por outro insumo não incluído na análise.

Para eliminar esse problema da produtividade parcial é que se calcula a produtividade total, que é a razão entre a soma ponderada dos produtos gerados pelos recursos consumidos. O problema está na correta seleção de variáveis que represente adequadamente o processo em estudo e atenda aos objetivos da avaliação. Outro aspecto a ser ressaltado é a questão da atribuição de pesos (preços) a cada uma dessas variáveis.

Sobre esses dois problemas, Lovell 2 relata que, se todos os produtos e insumos envolvidos no processo produtivo fossem incluídos na avaliação de produtividade total, o valor desta medida seria sempre 1. Frente a essa conclusão, o autor propôs definir produtividade como a razão entre a produção útil e o consumo útil, calculada na forma da equação 2

$$
P R=\frac{\sum \mu_{m} u_{m}}{\sum v_{n} x_{n}}(2)
$$

na qual: $u_{m} \geq 0$ é a quantidade gerada do produto $\mathrm{m}$, com $\Sigma u_{m}>0 ; \chi_{n} \geq 0$ (quantidade consumida do insumo $\mathrm{n}$ ), com $\Sigma_{n}>0 ; u_{m} \geq 0$ (utilidade do produto $\mathrm{m}$ na composição da produção útil); e $v_{n} \geq 0$ (utilidade do insumo $\mathrm{n}$ na composição do consumo útil).

Esse autor sugere que, na prática econômica, os preços virtuais $\mu_{m}$ e $v_{n}$ sejam representados pelos preços de mercado. A dificuldade de aplicação desse conceito está no fato de nem sempre se dispor dos preços, não serem confiáveis 


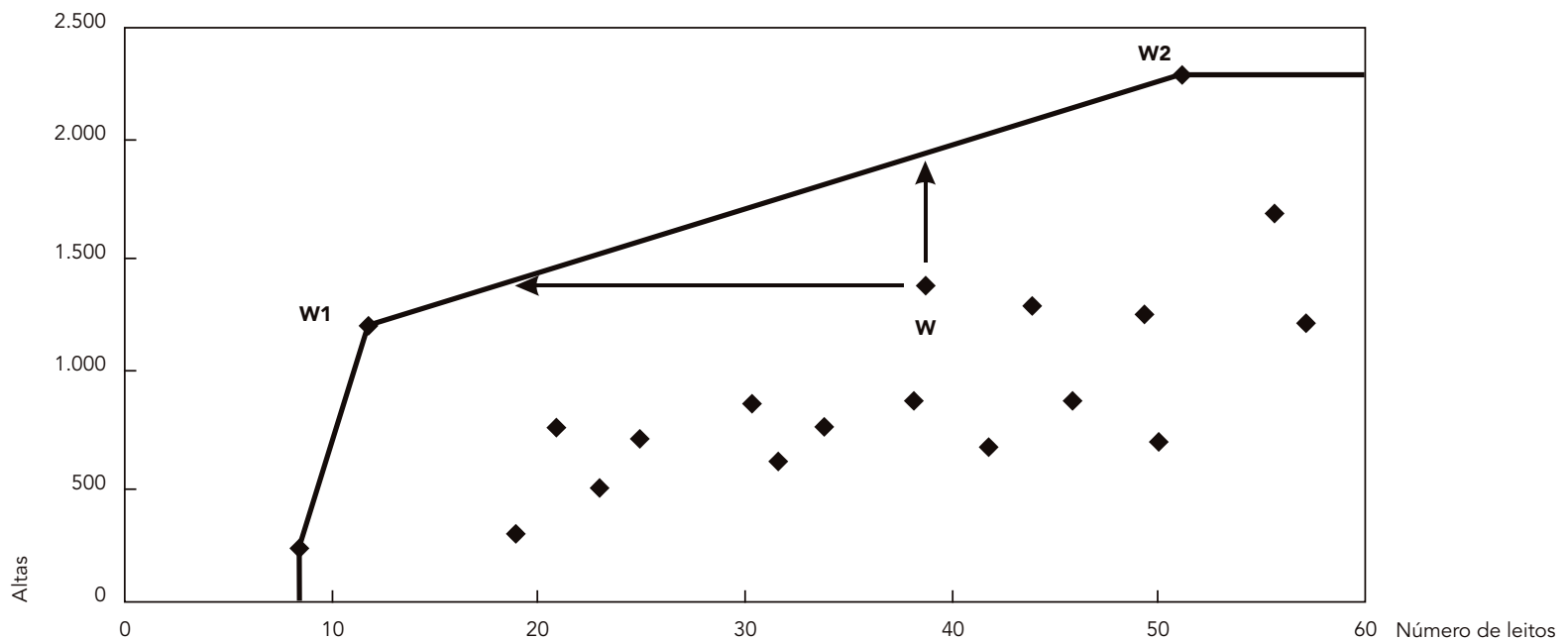

os valores, ou mesmo não existir preço de algum produto ou insumo.

A eficiência técnica diz respeito à comparação entre a produtividade do plano de operação executado por uma organização e a máxima produtividade que esta organização pode alcançar (máxima produtividade observada). Para operações que envolvem o emprego de múltiplos insumos na geração de múltiplos produtos, o conceito atualmente empregado de eficiência tem origem nos trabalhos de Pareto ${ }^{3}$, que propôs o bem-estar geral como critério para julgamento de qualquer política social. Dessa forma, aproximar-se de eficiência na alocação de recursos significa chegar o mais próximo possível da satisfação das necessidades individuais das pessoas, dadas as restrições dos recursos e da tecnologia produtiva vigente. Logo, uma alocação de recursos seria Pareto-eficiente se não fosse possível aumentar o bem-estar de uma pessoa sem diminuir o bem-estar de outra, dadas as restrições de recursos e tecnologia existentes.

Koopmans propôs em 1951 uma especialização microeconômica do conceito de eficiência de Pareto, sob a ótica da produção de bens e serviços gerada por uma organização. Esse autor ressalta que uma firma é eficiente do ponto de vista produtivo quando ela otimiza (maximiza) a produção gerada comparativamente ao consumo necessário. Esse tipo de eficiência é conhecido na literatura como eficiência produtiva Pareto-Koopmans 4.
Os estudos sobre eficiência produtiva ParetoKoopmans costumam considerá-la formada de duas componentes:

- A eficiência técnica ou física, que se refere à habilidade de evitar desperdícios, seja na utilização de uma quantidade de insumos maior que o necessário, seja na produção de bens ou serviços em quantidades inferiores às consideradas possíveis; e

- A eficiência econômica ou alocativa, que se refere à habilidade de uma organização maximizar a receita e minimizar custos e que, portanto, envolve as quantidades e os preços dos insumos consumidos e dos produtos gerados.

Marinho \& Façanha 5 salientam as dificuldades em analisar a eficiência alocativa em setores de difícil determinação de preço. Igualmente, reforçam a indicação da avaliação da eficiência técnica ao considerar que "as magnitudes de eventuais ineficiências alocativas são, em geral, menores do que as de eventuais ineficiências técnicas em um dado sistema".

Dessa forma, o objetivo deste estudo foi identificar quais os hospitais da rede hospitalar de Santa Catarina que são eficientes quanto ao aproveitamento de seus recursos e quanto é possível aumentar a produção dos hospitais ineficientes. A rede hospitalar catarinense foi selecionada para o estudo por constituir um grupo homogêneo de unidades em número suficiente para a realização da análise prevista. 
Para identificar os hospitais eficientes quanto ao aproveitamento de seus recursos foi empregada a Análise Envoltória de Dados (DEA; Data Envelopment Analysis), que é uma metodologia a qual emprega modelos de programação linear para construir fronteiras empíricas de eficiência produtiva. Esse estudo permite demonstrar que a abordagem DEA pode identificar os hospitais eficientes e realizar apontamentos para que os hospitais abaixo da fronteira empírica de produtividade otimizem a utilização dos recursos empregados. Foi feita aplicação de dados secundários da rede hospitalar de Santa Catarina para o ano de 2003, disponíveis no Sistema de Informações Hospitalares do SUS (SIH-SUS) do DATASUS.

\section{Metodologia}

A abordagem DEA é habitualmente utilizada para avaliar a eficiência de um conjunto de produtores. É um modelo em que cada "produtor", neste caso hospital, é comparado unicamente com o "melhor produtor". O pressuposto básico para a aplicação de DEA é a possibilidade de comparação entre organizações que fazem parte de um mesmo conjunto de referência, constituído pelas organizações que produzem sob uma mesma tecnologia e podem ter suas produtividades comparadas.

A abordagem DEA teve sua origem na medida CCR, desenvolvida por Charnes et al. ${ }^{6}$, em 1978, para avaliar entidades não lucrativas e programas públicos. A medida CCR, iniciais dos autores, aplica-se a tecnologias com retorno de escala constante (Constant Returns to Scale - CRS). Entretanto, essa primeira medida proposta carecia de ajustes para atender a outras hipóteses e restrições relativas ao conceito de produtividade.

Uma das mais representativas extensões à medida CCR foi proposta por Banker et al. 7, em 1984, com a apresentação da medida BCC, que é adequada para medir eficiência técnica em tecnologias que exibem retornos variáveis de escala.

O modelo BCC permite decompor a ineficiência técnica avaliada com o modelo CCR em duas componentes: a ineficiência de escala, associada a variações da produtividade decorrentes de mudanças na escala de produção, e a ineficiência de gestão, associada à habilidade gerencial da organização. A medida BCC indica a ineficiência de gestão. A ineficiência de escala é a razão entre o valor da medida BCC e o da medida CCR e indica a restrição que o porte da unidade avaliada faz ao aumento da produtividade.
Devido à sua interdisciplinaridade de aplicação em diferentes setores da economia, sua utilização teve um crescimento bastante acentuado. Esse modelo tem sido aplicado em saúde pública (clínicas e hospitais) 8,9,10, universidades, escolas, bancos, restaurantes e indústrias.

O método DEA tem as seguintes características que devem ser destacadas:

- Difere dos métodos baseados em avaliação puramente econômica, que necessitam converter todos os insumos e produtos em unidades monetárias;

- Os índices de eficiência são baseados em dados reais, e não em fórmulas teóricas;

- É uma alternativa e um complemento aos métodos da análise de tendência central e de custo benefício;

- Considera a possibilidade de que os valores discrepantes não representem apenas desvios em relação ao comportamento "médio", mas possíveis padrões de comparação a serem estudados pelas demais DMU (Decision Making Units).

Ao contrário das abordagens paramétricas tradicionais, o DEA otimiza cada observação individual com o objetivo de determinar uma fronteira linear por partes ("piece-wise linear") que compreende o conjunto de DMU Pareto-Eficiente, que são as unidades consideradas eficientes e não apresentam nenhuma folga de insumo ou de produto.

A Análise Envoltória de Dados foi concebida para ser aplicada a setores onde os produtos não são comparáveis em valores monetários, e é indicada em situações em que é necessária a conciliação de múltiplas variáveis de natureza e magnitude diversas, qualitativas e quantitativas, em um conjunto de elementos submetidos a diferentes realidades, sem um padrão preestabelecido.

\section{Modelo teórico de hospital}

De acordo com Slack 11, do ponto de vista produtivo o hospital pode ser descrito como tendo todos os elementos necessários, quais sejam:

- Entrada no sistema: pacientes;

- Recursos utilizados: estrutura física, recursos materiais, humanos e financeiros;

- Saída do sistema: pacientes após passar pelo processo.

Segundo Espigares 12, pode-se sistematizar o funcionamento de uma organização hospitalar da seguinte forma:

- Aplicação de Inputs (pessoal, equipamento, material sanitário etc.);

- Obtenção de produtos intermediários (radiografias, exames, alimentação); 
- Obtenção de produto final (realização de um parto, tratamento de uma doença);

- Obtenção de resultados (melhora do nível de saúde).

O hospital pode ser considerado uma empresa de múltiplos produtos onde cada um destes é composto de múltiplos bens e serviços. A definição de um "produto hospitalar" é um grande avanço para que se tenha uma gestão eficiente dos recursos financeiros.

O presente estudo adota, dentre as atividades de assistência, a internação como proxie de hospital, por se tratar de atividade típica deste tipo de instituição. O modelo teórico de hospital a ser empregado, representado pela função de internação, é apresentado na Figura 2.

\section{Modelo empírico de hospital}

Para o modelo empírico de hospital buscaram-se variáveis representativas para recursos humanos, recursos materiais e recursos financeiros para a representação dos insumos, bem como variáveis representativas para o produto hospitalar.

O produto da internação hospitalar dessa aplicação foi o número de altas geradas pelo hospital para pacientes do SUS. A variável utilizada para representar os recursos humanos foi o número de médicos somado ao número de profissionais da equipe auxiliar de enfermagem, haja vista que esta informação representou a maior correlação, significativa em nível de 5\%, com o produto empregado no modelo. Essas in-

Figura 2

Modelo teórico de hospital segundo a função de internação.

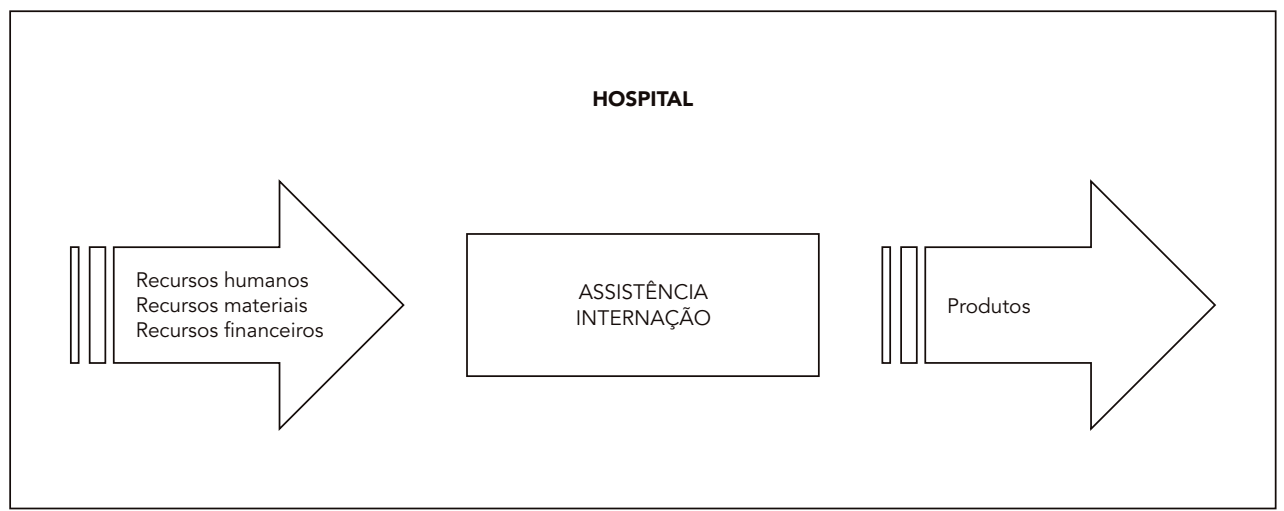

formações foram obtidas por meio do cadastro de profissionais por estabelecimento de saúde no DATASUS, sendo selecionados apenas os profissionais registrados no SUS. Para representar os recursos materiais foi adotada a variável "número de leitos conveniados ao SUS”, obtida no cadastro dos hospitais para o ano de 2003. Para garantir homogeneidade entre as unidades avaliadas, foram incluídos apenas os hospitais com mais de $80 \%$ dos leitos conveniados ao SUS. Esse corte foi empírico, no quartil inferior de número de leitos contratados, o que proporcionou a exclusão de $25 \%$ dos hospitais da rede com menor percentual de leitos conveniados ao SUS. Os recursos financeiros foram representados pela variável "valor total de AIH”, obtida nos arquivos reduzidos das Autorizações de Internação Hospitalar (AIH). Realizou-se inicialmente a estatística descritiva do banco de dados previamente construído com o objetivo de classificar e agrupar os hospitais do estudo.

\section{A definição do banco de dados}

Todos os hospitais brasileiros que prestam serviços ao SUS estão cadastrados no SIH-SUS, o qual é mensalmente atualizado. Esse sistema disponibiliza informações, tais como a localização do hospital, a natureza administrativa, o tempo de convênio, o tamanho e a distribuição da estrutu- 
ra física, os equipamentos, a quantidade e o tipo de leitos por especialidade, o número e o tipo de consultórios e de salas de atendimento, o tipo de gestão do sistema municipal, e a habilitação para complexidade por tipo de procedimento.

As atividades desenvolvidas ao longo das internações hospitalares são registradas no SIH-SUS, respeitando-se as normas estabelecidas para tal. A maior parte dos dados disponíveis no sistema de informações é extraída das AIH, documento emitido em função da solicitação de internação de paciente pelo SUS. Por meio da AIH pode-se identificar o paciente e os serviços prestados sob regime de internação hospitalar e fornecer informações para o gerenciamento do SUS. É por meio da AIH que hospitais, profissionais e serviços auxiliares de diagnose e terapia (SADT) se habilitam a receber pelos serviços prestados.

Foram extraídos arquivos reduzidos de AIH dos 12 meses de 2003 para o Estado de Santa Catarina. Os arquivos foram descompactados em um programa computacional - "expanddbf" - de livre distribuição pelo DATASUS. Com os arquivos em formato "dbf", os mesmos foram transportados para o programa Epi Info versão 6.04 (Centers for Disease Control and Prevention, Atlanta, Estados Unidos) e os 12 arquivos foram agrupados em uma única planilha de dados onde cada uma das colunas corresponde a uma variável extraída da AIH, e cada linha corresponde a um número de guia de AIH.

Também foram extraídos os arquivos de cadastro de hospitais para os 12 meses do ano de 2003. Essa etapa teve como objetivo a obtenção do número total de leitos e do número de leitos conveniados ao SUS.

As informações sobre o número de médicos e equipe de enfermagem, representadas pelo número de auxiliares de enfermagem, número de atendentes de enfermagem e número de técnicos de enfermagem, por estabelecimento (hospital), foram obtidas na base de dados do Cadastro Nacional de Estabelecimentos de Saúde (CNES). A soma do número de médicos com o número de profissionais na equipe auxiliar de enfermagem representou os recursos humanos dos hospitais.

Os hospitais foram agrupados em categorias com características de porte e especificidade semelhantes tendo em vista a indicação da abordagem DEA. Foram selecionados os hospitais gerais e excluídos os especializados, pois a fronteira de eficiência deve ser formada por unidades semelhantes quanto aos insumos e produtos de que dispõe no seu processo produtivo. Foram calculadas as taxas de internação por especialidade a fim de se identificar hospitais com maior nível de especialização em determinada área de atendimento médico.

Os hospitais cuja atuação é exclusivamente especializada-obstetrícia, psiquiatria, pediatria, ortopedia, cardiologia, neurologia etc. - foram excluídos na primeira análise do cadastro. Entretanto, alguns hospitais não são denominados especializados embora possuam determinada especificidade de atuação. Para identificá-los foi utilizada a taxa de internação por especialidades, aplicando-se um corte estatístico no percentil 95 em cada uma delas. Os hospitais com número de internações superiores ao percentil 95 em cada uma das especialidades foram considerados especializados e foram excluídos da análise.

Ao se analisar os arquivos reduzidos de $\mathrm{AIH}$, constataram-se alguns hospitais que não repassaram suas informações nos 12 meses do ano de 2003; tendo em vista a fidedignidade dos resultados optou-se por excluir do banco de dados os hospitais que deixaram de registrar as AIH's por três ou mais meses, bem como os hospitais que não registraram AIH's por dois meses consecutivos. Essas exclusões foram fundamentadas na observação da série histórica dos valores de AIH. Nos dados de 2003 para a rede catarinense observou-se que, quando um hospital não fazia sua cobrança em um determinado mês, em geral, lançava estes valores acumulados com os do mês seguinte. Todavia, quando isso se repetia por dois meses consecutivos ficava comprometida a análise dos valores lançados no terceiro mês por conterem o acúmulo dos dois anteriores. Da mesma forma, a falta de registro por três meses intercalados também prejudicava essa análise da série histórica dos valores.

Dos hospitais que permaneceram no banco de dados do estudo, verificou-se quais estavam cadastrados no Sistema Integrado de Procedimentos de Alta Complexidade (SIPAC). Hospitais cadastrados em um ou mais tipos de procedimentos foram excluídos, excetuando-se os códigos 190 (esterilização) e 380 (hospital-dia/cirurgia). A exclusão dos hospitais cadastrados no SIPAC deu-se para garantir que não houvesse grande quantidade de procedimentos de maior complexidade em algumas unidades analisadas, pois isto implicaria valores diferenciados de internações para exames e diagnósticos especializados.

Os hospitais universitários não fazem parte do estudo, pois este tipo de hospital tem, além da função de assistência, a função de ensino (formação profissional). Também foram excluídos os hospitais com taxas de internação de longa permanência maiores do que $10 \%$.

Observados os critérios de inclusão e exclusão, a amostra permaneceu com 112 hospitais, dos quais se possuía a totalidade das informa- 
ções. A estatística básica desse grupo de hospitais é apresentada na Tabela 1. Ressalte-se que a análise da distribuição de valores observados não denunciou a presença de outliers ou valores extremos.

A abordagem DEA preconiza que os planos de operação observados devem pertencer a uma mesma tecnologia produtiva, justificando o fato de se analisar apenas os hospitais gerais. Os modelos DEA que foram desenvolvidos neste estudo são genéricos, aplicáveis a qualquer rede de hospitais, e estão indicados para a avaliação da eficiência hospitalar, do ponto de vista do gestor do sistema de saúde. Primeiramente, empregou-

\begin{tabular}{|c|c|c|c|c|c|c|c|c|}
\hline \multirow[t]{2}{*}{ Variáveis } & \multicolumn{8}{|c|}{ Estatísticas básicas } \\
\hline & Média & $\begin{array}{l}\text { Desvio- } \\
\text { padrão }\end{array}$ & $\begin{array}{l}\text { CVP } \\
(\%)\end{array}$ & Mínimo & 10 quartil & Mediana & 3o quartil & Máximo \\
\hline Médicos e equipe de enfermagem & 36,2 & 36,5 & 101 & 4,0 & 14,0 & 21,5 & 46,3 & 231,0 \\
\hline Leitos do SUS & 43,4 & 26,8 & 62 & 13,8 & 26,8 & 35,4 & 52,8 & 158,2 \\
\hline Valor total das AlH (em mil Reais) & 338,6 & 375,2 & 111 & 38,4 & 38,4 & 206,5 & 376,0 & $1.916,1$ \\
\hline Total de altas & $1.050,8$ & $1.017,4$ & 97 & 154,0 & 154,0 & 676,5 & $1.334,0$ & $5.415,0$ \\
\hline
\end{tabular}

AlH: Autorizações de Internação Hospitalar; CVP: Coeficiente de Variação de Pearson.

se o modelo DEA-BCC orientado para a produção, considerando que o gestor do SUS avalia a direção de cada hospital por sua habilidade de maximizar o número de internações (que visam ao diagnóstico e ao tratamento de pacientes), dados os recursos acordados. Após, foi aplicado o modelo DEA-BCC orientado para a redução de consumo, considerando que o gestor do SUS também avalia a direção dos hospitais quanto aos recursos que está consumindo em excesso, ou seja, quanto poderia reduzir em número de leitos, pessoal e valor de AIH para produzir as mesmas altas.

\section{Resultados e discussão}

Marinho 13 menciona que hospitais costumam possuir alguma capacidade ociosa para permitir as adequações necessárias nos casos de imprevistos, excessos de demanda, e impossibilidades de transferências. Tal característica dificulta ajustes de curto prazo e indicaria a adoção da hipótese de retornos constantes de escala no planejamento organizacional.

No entanto, há evidências empíricas que, para a rede hospitalar de Santa Catarina, de acordo com os dados de 2003, não são constantes os retornos a mudanças na escala de operação destas instituições, haja vista que as produti- vidades parciais são decrescentes com o porte dos hospitais. Frainer 14 constatou que o modelo DEA-BCC é mais apropriado que o modelo DEA-CCR para a avaliação de eficiência em hospitais universitários, e constatação similar foi feita por Wolff 15 para os hospitais filantrópicos e privados em Santa Catarina.

De acordo com a abordagem DEA, um hospital ineficiente pode tornar-se eficiente ao se projetar para um dado ponto (X0,Y0) na superfície da fronteira de produção definida pela programação linear.

O programa linear para o modelo DEA-BCC orientado para a produção pode assim ser escrito na sua forma de envelopamento:

$F_{B C C}^{*}=\max \left\{\phi+\varepsilon\left(\sum t_{m}+\sum s_{n}\right)\right\}$

Sujeito a $\sum z_{j} x_{j n}+s_{n}=x_{0 n} \quad n=1,2, \ldots, N$

$\phi \cdot u_{0 m}-\sum z_{j} u_{j m}+t_{m}=0 \quad m=1,2, \ldots, M$

$\sum z_{j}=1$ (retorno variável à escala de produção)

$z_{j} \geq 0 \quad j=1,2, \ldots J$

$t_{m} \geq 0 \quad m=1,2, \ldots M$

$s_{n} \geq 0 \quad n=1,2, \ldots N$

$\varepsilon>0$ não-arquimediano

$\Sigma z_{j} \geq=1$ indica retorno variável à escala de produção;

$t_{m} \geq 0$ representa as folgas nos produtos;

$s_{n} \geq 0$ representa as folgas nos insumos. 
Esse modelo de programação linear proposto encontrou 23 hospitais eficientes (Tabela 2).

Pode-se observar que, dentre os hospitais avaliados, e de acordo com o modelo empregado, a maioria dos hospitais eficientes é filantrópico e de pequeno porte. Porém, não houve associação entre eficiência e natureza administrativa ou porte do hospital. Isso ocorreu em conseqüência da característica da amostra, formada por $65 \%$ de hospitais filantrópicos e $71 \%$ de hospitais de pequeno porte.

Os resultados indicam que o número de altas poderia ser aumentado em $15 \%$ caso os recursos fossem utilizados de maneira eficiente pela rede hospitalar em estudo, ou seja, poderiam ser geradas, em termos de valores projetados segundo a abordagem DEA, 135 mil altas em vez das $118 \mathrm{mil}$ geradas em 2003.

Ao se aplicar o modelo DEA orientado para a redução de insumos, o número de médicos, técnicos de enfermagem e auxiliares de enfermagem poderia ser reduzido em $25 \%$, isto implica uma redução de mais de mil profissionais, passando de 4.048 para 3.036. Para o número de leitos seria possível uma redução de $17 \%$, ou seja, os leitos da rede em estudo passariam de 4.871 para 4.043. E os valores de AIH poderiam ser reduzidos em 13\%, de cerca de 38 milhões de Reais para aproximadamente 33 milhões de Reais. A Tabela 3 apresenta as reduções percentuais em cada insumo e a expansão da produção segundo porte e natureza administrativa dos hospitais.

Este estudo não buscou comparar médias de escores de eficiência entre os hospitais públicos e privados, pois a amostra analisada possui apenas quatro hospitais públicos. Entretanto, mui- tos estudos utilizam DEA para tal comparação de escores de eficiência entre públicos e privados. Rebba \& Rizzi 16 avaliaram a eficiência de 85 hospitais na região de Vêneto na Itália e apontaram que os escores de eficiência nos hospitais públicos são ligeiramente melhores que nos privados. Os hospitais privados envolvidos no estudo são responsáveis por internações de longa permanência, explicando em parte a diferença de escore entre hospitais públicos e privados detectada pelos autores.

No Brasil, Calvo 1 avaliou a eficiência de 40 hospitais públicos e 40 privados no Estado do Mato Grosso, a fim de saber se havia associação entre natureza administrativa e eficiência. O estudo identificou 12 hospitais públicos e 14 privados eficientes, sem diferença significativa entre as proporções, sugerindo que a gestão nos dois tipos de hospitais, segundo a eficiência produtiva, é semelhante.

Marinho 17,18 tem desenvolvido diversos estudos com aplicação de DEA em saúde, identificando as diferentes possibilidades de utilização da abordagem. Para esse autor, um aspecto que merece destaque é que a simples identificação de uma unidade eficiente não garante que esta seja modelo de gestão. Cabe observar a freqüência dessa unidade como referência. As unidades mais freqüentes como referência podem oferecer bons indícios de aprimoramento da gestão; as unidades menos freqüentes podem indicar uma especialização no conjunto.

Os 23 hospitais eficientes na aplicação realizada foram pares de referência para pelo menos um hospital. Sete hospitais foram pares de referência para no máximo cinco hospitais; outros

Tabela 2

Número e percentual de hospitais eficientes e ineficientes segundo a natureza administrativa e o porte.

\begin{tabular}{|c|c|c|c|c|c|c|}
\hline \multirow[t]{2}{*}{ Hospital } & \multicolumn{2}{|c|}{ Eficientes } & \multicolumn{2}{|c|}{ Ineficientes } & \multicolumn{2}{|c|}{ Total } \\
\hline & $\mathrm{n}$ & $\%$ & $\mathbf{n}$ & $\%$ & $\mathbf{n}$ & $\%$ \\
\hline \multicolumn{7}{|c|}{ Natureza administrativa } \\
\hline Contratado & 8 & 23 & 27 & 77 & 35 & 100 \\
\hline Filantrópico & 14 & 19 & 59 & 81 & 73 & 100 \\
\hline Municipal & 1 & 25 & 3 & 75 & 4 & 100 \\
\hline Total & 23 & 21 & 89 & 79 & 112 & 100 \\
\hline \multicolumn{7}{|c|}{ Porte (número de leitos) } \\
\hline $151-500$ & 1 & 100 & - & 0 & 1 & 100 \\
\hline $51-150$ & 7 & 23 & 24 & 77 & 31 & 100 \\
\hline$\geq 50$ & 15 & 19 & 65 & 81 & 80 & 100 \\
\hline Total & 23 & 21 & 89 & 79 & 112 & 100 \\
\hline
\end{tabular}


Redução percentual de insumos e expansão da produção para modelo DEA-BCC, segundo porte e natureza administrativa dos hospitais.

\begin{tabular}{|c|c|c|c|c|}
\hline \multirow[t]{2}{*}{ Hospital } & \multicolumn{3}{|c|}{ Redução de insumos (\%) } & \multirow{2}{*}{$\begin{array}{c}\text { Expansão da } \\
\text { produção (\%) } \\
\text { Altas }\end{array}$} \\
\hline & Médicos e equipe & Leitos & Valor das AlH & \\
\hline \multicolumn{5}{|c|}{ Natureza administrativa } \\
\hline Contratado & 29 & 19 & 16 & 20 \\
\hline Filantrópico & 23 & 17 & 13 & 14 \\
\hline Municipal & 25 & 10 & 10 & 17 \\
\hline \multicolumn{5}{|c|}{ Porte (número de leitos) } \\
\hline $51-150$ & 28 & 18 & 14 & 13 \\
\hline$\geq 50$ & 23 & 18 & 13 & 20 \\
\hline
\end{tabular}

AlH: Autorizações de Internação Hospitalar.

sete foram pares de referência para mais do que 15 hospitais; dois pequenos - um contratado e outro filantrópico - foram pares de referência para mais do que sessenta hospitais. Esses dois últimos podem ser observados como exemplos para melhorar a gestão da maioria dos hospitais analisados.

As variáveis sócio-econômicas e as que refletem o perfil de morbidade da população não foram controladas neste estudo, mas a pesquisa de Wolff 15 desenvolveu um modelo para avaliar o impacto do ambiente operacional na produtividade dos hospitais brasileiros. A aplicação foi realizada em 74 hospitais privados e filantrópicos em Santa Catarina para o ano de 2002. Nesse estudo, a exemplo do apresentado em outras aplicações da abordagem DEA 5,13, a autora utilizou regressão censurada para analisar os escores de eficiência do DEA em relação às outras variáveis. Os fatores ambientais identificados como agentes de alteração na produtividade dessas instituições foram:

- As características da clientela - suas condições gerais de saúde;

- As condições de saneamento básico do município; e

- A cobertura de serviços básicos de atenção à saúde da população do município.

A pesquisa não obteve evidências de que o fator econômico - participação desses hospitais no mercado do SUS em Santa Catarina, nem tampouco que fatores sócio-econômicos (escolaridade, esperança de vida ao nascer e renda per capita) e demográficos (\% de pessoas idosas na população) afetam a produtividade hospitalar, sendo que a autora recomenda estudos mais aprofundados para averiguação destas questões.

\section{Conclusões}

A questão central deste estudo foi identificar os hospitais eficientes, pertencentes à rede hospitalar de Santa Catarina, quanto ao aproveitamento de seus recursos. Buscou-se também determinar quanto se pode aumentar a produção da rede em estudo, segundo o modelo empregado.

Constatou-se que os retornos são variáveis à escala de produção e aplicou-se o modelo DEABCC. Contemplar o retorno variável à escala de produção é uma das vantagens da abordagem DEA sobre técnicas de produtividade parcial, as quais não consideram o efeito do porte do hospital na sua produtividade, apesar de ser conhecida a característica de redução relativa dos níveis de produtividade para unidades produtoras de maior porte em diferentes setores produtivos, inclusive o hospitalar.

A Análise Envoltória de Dados permite estimar a composição ideal entre múltiplos insumos e múltiplos produtos, indicando qual a composição ideal para cada hospital, como foi mostrado neste estudo com três insumos e um produto.

O recorte desta aplicação foi para a assistência hospitalar - especialmente para a internação - em hospitais gerais, com amostra dos hospitais conveniados ao SUS em Santa Catarina. O estudo teve a finalidade de demonstrar o potencial da abordagem DEA para análises de eficiência produtiva na saúde, a exemplo de outras aplicações desenvolvidas no Brasil, principalmente com base no enfoque econômico. Apesar disso, cabe reforçar que o modelo adotado é genérico e pode ser aplicado a qualquer outro grupo de hospitais, desde que observadas as restrições e características da abordagem. 


\section{Resumo}

O presente trabalho avaliou a eficiência produtiva de 112 hospitais conveniados ao Sistema Único de Saúde (SUS) no Estado de Santa Catarina, Brasil, com o objetivo de verificar quais são os hospitais eficientes quanto ao aproveitamento de seus recursos. Os dados do estudo são de 2003, obtidos por meio do Sistema de Informações Hospitalares do SUS (SIH-SUS). Aplicouse a abordagem DEA (Data Envelopment Analysis) para retornos variáveis às mudanças na escala de produção (modelo DEA-BCC). A abordagem preconizou a avaliação de hospitais gerais com características de especificidade semelhantes. Os resultados do estudo indicaram 23 hospitais eficientes, além de apontar as metas eficientes de produção para cada unidade avaliada. De acordo com o modelo empírico definido, o número de internações com altas poderia ser aumentado em $15 \%$ de acordo com o modelo DEA orientado para expansão da produção. Quando se aplica o modelo DEA orientado para a redução de insumos, o número de médicos, técnicos de enfermagem e auxiliares de enfermagem poderia ser reduzido em $25 \%$, o número de leitos em $17 \%$ e o valor de AIH em 13\% para a rede hospitalar em estudo.

Avaliação em Saúde; Eficiência Organizacional; Avaliação de Serviços de Saúde

\section{Colaboradores}

A. Cesconetto participou da redação e revisão do artigo e foi o responsável pela coleta e análise dos dados e aplicação do modelo DEA. J. S. Lapa participou da revisão do artigo e foi responsável pelas definições teóricas e metodológicas do modelo DEA adotado. M. C. M. Calvo definiu a amostra de hospitais, indicou a metodologia a ser empregada (análise envoltória de dados), participou da análise dos dados, redação e revisão final do artigo.

\section{Referências}

1. Calvo MCM. Hospitais públicos e privados no Brasil: o mito da eficiência produtiva no Estado de Mato Grosso em 1998 [Tese de Doutorado]. Florianópolis: Programa de Pós-graduação em Engenharia de Produção, Universidade Federal de Santa Catarina; 2002.

2. Lovell CAK, Schmidt SS. The measurement of productive efficiency: techniques and applications. New York: Oxford University Press; 1993. p. 3-67.

3. Pareto V. Coleção grandes cientistas sociais. São Paulo: Editora Ática; 1984.

4. França JMF. Um modelo para avaliar o impacto da assimetria de informação na gestão de organizações sociais com aplicação às universidades federais brasileiras [Tese de Doutorado]. Florianópolis: Programa de Pós-graduação em Engenharia de Produção, Universidade Federal de Santa Catarina; 2005.
5. Marinho A, Façanha LO. Hospitais universitários: avaliação comparativa de eficiência técnica. Economia Aplicada 2000; 4:315-49.

6. Charnes A, Cooper WW, Rhodes E. Measuring the efficiency of decision-making units. Eur J Oper Res 1978; 3:339.

7. Banker RD, Charnes A, Cooper WW. Some models for estimation technical and scale inefficiency in data envelopment analysis. Manage Sci 1984; 30:1078-92.

8. Giuffrida A, Gravelle H. Measuring performance in primary care: econometric analysis and DEA. Heslington: Department of Economics and Related Studies, University of York; 1999.

9. Retzlaff-Roberts D, Chang CF, Rubin RM. Technical efficiency in the use of health care resources: a comparison of OECD countries. Health Policy 2004; 69:55-72. 
10. Pinillos M, Antoñanzas F. La atención primaria de salud: descentralización y eficiencia. Gac Sanit 2002; 16:401-7.

11. Slack N, Chambers S, Johnston R. Administração da produção. São Paulo: Editora Atlas; 2002.

12. Espigares JLN. Análisis de la eficiencia en las organizaciones hospitalarias públicas. Granada: Editorial Universidad de Granada; 1999.

13. Marinho A. Avaliação da eficiência técnica nos serviços de saúde dos municípios do Estado do Rio de Janeiro. Revista Brasileira de Economia 2003; 57:515-34.

14. Frainer DM. A eficiência técnica de hospitais universitários federais brasileiros no primeiro semestre de 2001 [Dissertação de Mestrado]. Florianópolis: Programa de Pós-graduação em Engenharia de Produção, Universidade Federal de Santa Catarina; 2004.
15. Wolff LDG. Um modelo para avaliar o impacto do ambiente operacional na produtividade de hospitais brasileiros [Tese de Doutorado]. Florianópolis: Programa de Pós-graduação em Engenharia de Produção, Universidade Federal de Santa Catarina; 2005.

16. Rebba V, Rizzi D. The role of demand and weight restrictions in DEA measurement of hospital efficiency with an application to the hospitals of Veneto Region - Italy. Padova: Università degli Studi di Padova; 2003. (Discussion Papers).

17. Marinho A. Estudo de eficiência em alguns hospitais públicos e privados com a geração de rankings. Rio de Janeiro: Instituto de Pesquisa Econômica Aplicada; 2001. (Texto para Discussão, 794).

18. Marinho A. Hospitais universitários: indicadores de utilização e análise de eficiência. Rio de Janeiro: Instituto de Pesquisa Econômica Aplicada; 2001. (Texto para Discussão, 833).

Recebido em 20/Set/2006

Versão final reapresentada em 24/Jan/2008

Aprovado em 13/Mar/2008 